\title{
Multidisciplinary diagnostic and therapeutic approaches to pancreatic cystic lesions
}

This article was published in the following Dove Press journal:

Journal of Multidisciplinary Healthcare

3 February 2014

Number of times this article has been viewed

\author{
Michael J Clores \\ Amar Thosani \\ Jonathan M Buscaglia \\ Division of Gastroenterology, \\ Department of Medicine, Stony Brook \\ University School of Medicine, Stony \\ Brook, NY, USA
}

\begin{abstract}
Pancreatic cystic lesions are commonly encountered today with the routine use of cross-sectional imaging modalities such as computed tomography (CT) and magnetic resonance imaging (MRI). The majority of patients discovered to have a pancreatic cyst are completely asymptomatic; yet the presence of such a finding instills fear in the minds of both patient and physician, as the concern for malignant transformation to pancreatic cancer is great despite the relatively low overall likelihood of cyst progression. Not all cysts in the pancreas represent pancreatic cystic neoplasms (PCNs), and not all PCNs have significant malignant potential. Mucinous PCNs are the most concerning, as these lesions have the greatest potential for cancerous transformation to adenocarcinoma. Within the group of mucinous PCNs, intraductal papillary mucinous neoplasms (IPMNs) involving the main pancreatic duct are the most worrisome, and surgical resection should be pursued if the patient has appropriate operative risks. IPMN lesions involving the branch ducts, and mucinous cystadenomas, have a lower likelihood for malignancy, and they may be closely followed for the development of any worrisome or highrisk features. Surveillance of known PCNs is performed with a combination of CT, MRI and endoscopic ultrasound (EUS). EUS-guided fine-needle aspiration (EUS-FNA) may be used to assess cyst fluid cytology, and also to detect cyst fluid amylase level, carcinoembryonic antigen level, and DNA molecular analysis in certain cases. The presence or absence of specific cyst morphological features, as well as the cyst fluid analysis, is what enables the physician to guide the patient towards continued surveillance, versus the pursuit of surgical resection.
\end{abstract}

Keywords: endoscopic ultrasound, EUS-guided fine-needle aspiration, mucinous cystadenoma, intraductal papillary mucinous neoplasm, surveillance

\section{Introduction}

The diagnosis and management of pancreatic cystic lesions has become an area of developing interest over the past decade. Increasing use of advanced abdominal imaging modalities has resulted in the discovery of previously unrecognized pancreatic cystic neoplasms (PCNs). As these lesions have become a more common finding, health care providers should be familiar with the different types of cystic pancreatic lesions in order to assess the potential for malignancy within a cyst. This allows providers to effectively risk-stratify patients for surveillance, surgery, or expectant management. The purpose of this review is to provide both general practitioners and specialists with evidence-based data to aid in the management of patients found to have PCNs.

\section{Epidemiology and classification of pancreatic cysts}

Pancreatic cysts are classified as either a "true cyst" in which there is a true epithelial lining to the lesion; or a pseudocyst ("false cyst"), in which there is a walled-off collection 
Table I Types of pancreatic cysts

\begin{tabular}{|c|c|c|c|c|c|}
\hline \multirow[t]{2}{*}{ Characteristic } & \multicolumn{5}{|l|}{ Cyst type } \\
\hline & Pseudocyst & SCA & MCN & IPMN & SPN \\
\hline Age & Variable & Middle-aged & Middle-aged & Elderly & Young \\
\hline Sex & $M>F$ & $\mathrm{~F}>\mathrm{M}$ & $\mathrm{F}$ & $M=F$ & $\mathrm{~F}$ \\
\hline Pancreatitis history & Yes & No & No & Yes & No \\
\hline Location & Evenly & Evenly & Body/tail & Head & Evenly \\
\hline Malignant potential & None & Rarely & Moderate to high & Low to high & Low \\
\hline Biliary obstruction & Yes, uncommon & No & No & Yes, uncommon & No \\
\hline
\end{tabular}

Abbreviations: F, female; IPMN, intraductal papillary mucinous neoplasm; M, male; MCN, mucinous cystic neoplasm; SCA, serous cystadenoma; SPN, solid pseudopapillary neoplasm.

of fluid without a true epithelial lining, usually the result of acute pancreatitis. PCNs are true pancreatic cysts, and they represent at least $50 \%$ of all pancreatic cystic lesions.

PCNs are generally divided into four subtypes: mucinous cystic neoplasms (MCNs), intraductal papillary mucinous neoplasms (IPMNs), serous cystadenomas (SCAs), and solid pseudopapillary neoplasms (SPNs) (Table 1$){ }^{1-3}$ The MCN and IPMN types are further classified as mucin-producing lesions, while the remainder are non-mucinous. ${ }^{4}$ Differentiating between the different cyst types can be challenging; however, certain radiographic, histological, and pathological features may help distinguish these cysts from one another, and thus help guide management for the patient.

SCAs represent roughly $30 \%$ of PCNs. They occur more commonly in women, and their peak incidence is in the seventh decade of life. The cyst lining is composed of a simple, glycogen-rich cuboidal epithelium. On imaging, these cysts appear as honeycomb-like microcysts, often with the presence of a "central scar" on computed tomography (CT) or magnetic resonance imaging (MRI). The tiny microcystic spaces may often coalesce, and the lesion can begin to appear as a solid mass-like structure (Figure 1). The most common location is in the body and tail of the pancreas. These lesions

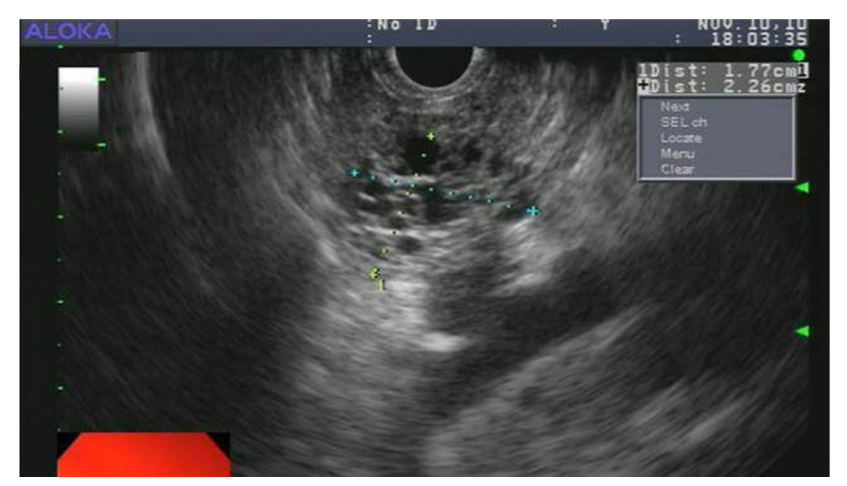

Figure I Endoscopic ultrasound image of a $2.26 \mathrm{~cm}$ serous cyst adenoma. Multiple small microcystic spaces coalesce to form a mass-like lesion in the body of the pancreas. are usually benign, with a very low potential for malignant transformation, and thus they are typically managed conservatively unless the patient is symptomatic from the cyst (eg, abdominal pain). ${ }^{3}$ However, cases of malignant transformation of a serous cystic lesion into a serous cystadenocarcinoma have been reported. In a retrospective review of 158 resection specimens of serous cystic pancreatic lesions from a single institution, one case of histologically confirmed malignancy was identified. ${ }^{5}$ Also, three of these cases were classified as locally aggressive benign lesions, one of which later developed metachronous metastatic lesions. Additionally, a literature review of serous cystic lesions in 2009 indicated that an average lesion size of $10 \mathrm{~cm}$ was associated with carcinoma. ${ }^{6}$ Therefore, consideration should be made to treat larger and locally advanced lesions aggressively.

A relationship between SCAs and von Hippel-Lindau (VHL) disease has been noted. In one study, a histopathological analysis of pancreatic cysts from nine VHL patients was performed. A total of 21 benign serous lesions, 63 microscopic microcystic (serous) adenomas, and 35 macroscopic microcystic (serous) adenomas were found. ${ }^{7}$ All lesions displayed similar histology, and deoxyribonucleic acid (DNA) extracted from the cysts showed allelic deletions in the VHL gene. Another study showed evidence of VHL gene alterations not only in VHL-disease-associated cysts, but also in sporadic microcystic (serous) adenomas; thus implying that changes in the VHL tumor suppressor gene play an important role in the pathogenesis of these types of cysts, regardless of whether or not the individual has VHL disease. ${ }^{8}$

MCNs comprise $10 \%-45 \%$ of PCNs, occur mostly in the female population, and are typically discovered in the fifth and sixth decades of life. The location is usually in the pancreatic body or tail. MCNs commonly exhibit macrocystic spaces with thin septations. The MCNs are histologically very similar to IPMNs, as both lesions have a mucin-producing epithelial lining. However, a distinguishing feature between MCNs and IPMNs is the characteristic histopathological dense 

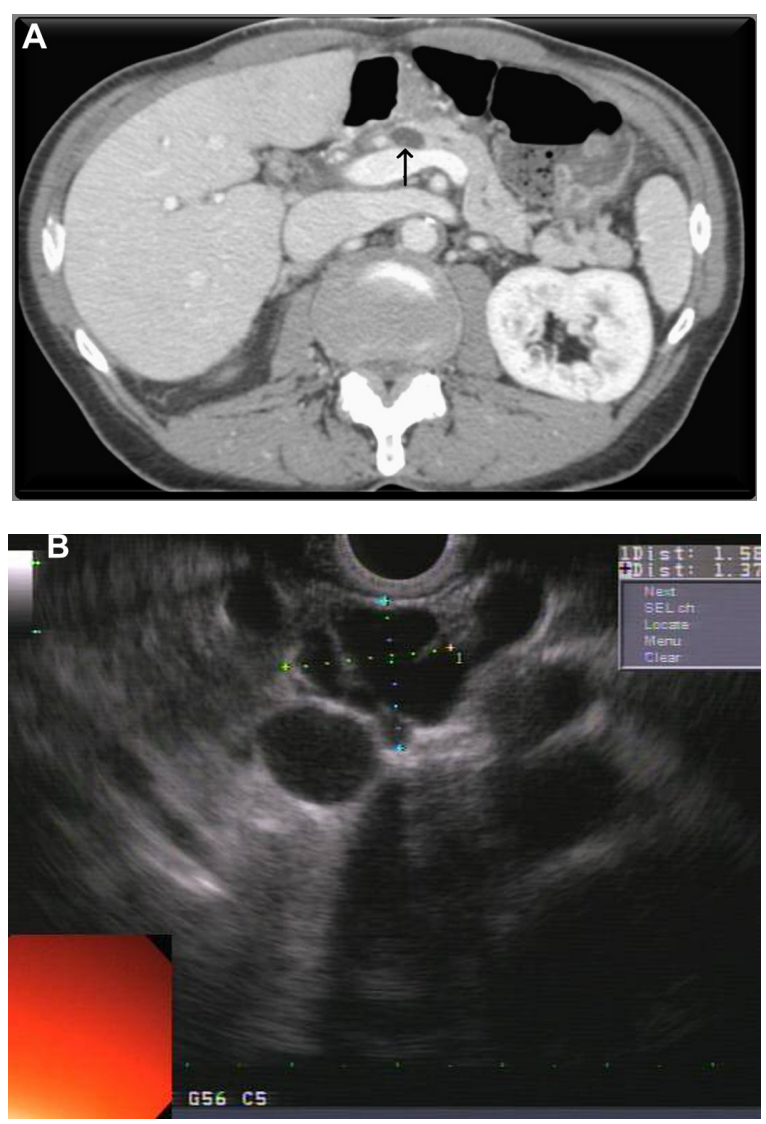

Figure 2 (A) Computed tomography scan with an incidentally found mucinous cystadenoma just inferior to the pancreatic duct in the neck of the gland (black arrow). Close inspection of the cysts suggests thick internal septations. (B) Endoscopic ultrasound image of a noncommunicating $1.6 \mathrm{~cm}$ mucinous cystadenoma lesion (dotted lines) with septations and macrocystic spaces.

mesenchymal "ovarian-like stroma" seen in MCNs. Also, MCNs do not communicate with the pancreatic ductal system, as they develop out in the periphery of the gland (Figure 2). All MCNs have a risk for malignant transformation, and therefore resection is generally considered in individuals who are good surgical candidates depending upon their clinical risk factors. ${ }^{3}$ The prevalence of invasive carcinoma in MCNs at the time of surgical resection varies from $6 \%$ to $36 \%{ }^{4}$ However, some studies have not used ovarian-type stroma as a necessary criterion to distinguish MCNs from IPMNs, making these data difficult to interpret. In some studies, the prevalence of invasive carcinoma strictly in MCNs with ovarian-type stroma ranges from $6 \%$ to $27 \%$. To avoid mistakenly classifying IPMNs as MCNs, which may have different clinical implications for the patient, the diagnosis of MCN should be limited to cysts containing ovarian-type stroma. ${ }^{4}$

IPMNs represent approximately $21 \%-33 \%$ of PCNs. IPMNs occur with equal frequency in both men and women, commonly in the sixth and seventh decades of life, and more often in the head of the pancreas. The cyst lining consists of a mucin-secreting columnar epithelium. A key feature of IPMN is communication with the pancreatic ductal system. Diffuse or segmental dilatation of the main or branch pancreatic ducts may be seen. All IPMNs have malignant potential, and similar to MCN lesions, an algorithm for risk-stratification and management is of paramount importance (discussed below). IPMNs with adenomatous or borderline changes have been shown to have an excellent prognosis if resected; however, the prognosis is less favorable when findings of carcinoma in situ or invasive carcinoma are present. ${ }^{3}$

IPMNs can be divided into three different subtypes: 1) main duct IPMN (MD-IPMN), involving dilation of the main pancreatic duct (MPD) only; 2) branch duct IPMN (BDIPMN), involving cystic dilation of one of the ductal sidebranches; and 3) mixed type, in which both the main duct and side-branch are involved in cystic dilation. These lesions are usually discovered on abdominal imaging studies. Segmental or diffuse dilatation of the MPD $>5 \mathrm{~mm}$, in the absence of other secondary causes such as chronic pancreatitis, is suggestive of MD-IPMN (Figure 3). Mucinous cysts communicating with the pancreatic ductal system without
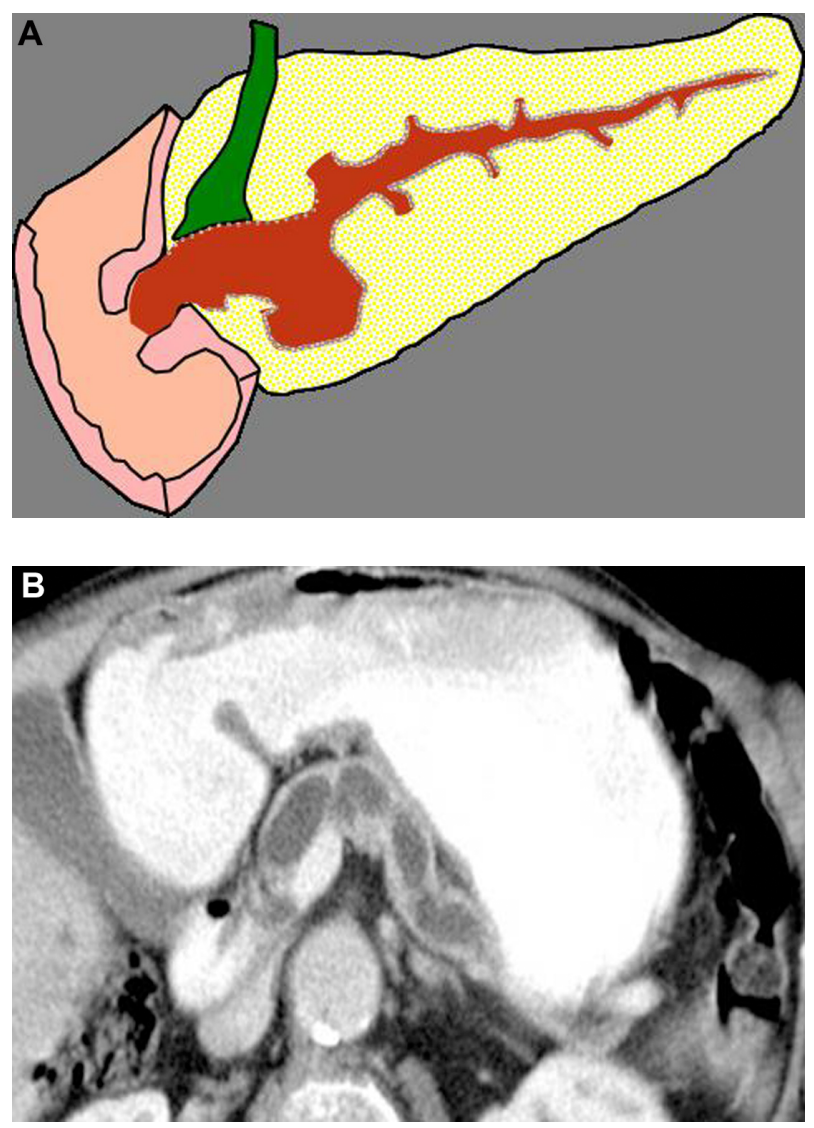

Figure 3 (A) Schematic of a main duct IPMN lesion, with diffuse involvement of the duct. (B) Magnetic resonance image of a diffusely involved main duct IPMN lesion. Abbreviation: IPMN, intraductal papillary mucinous neoplasm. 

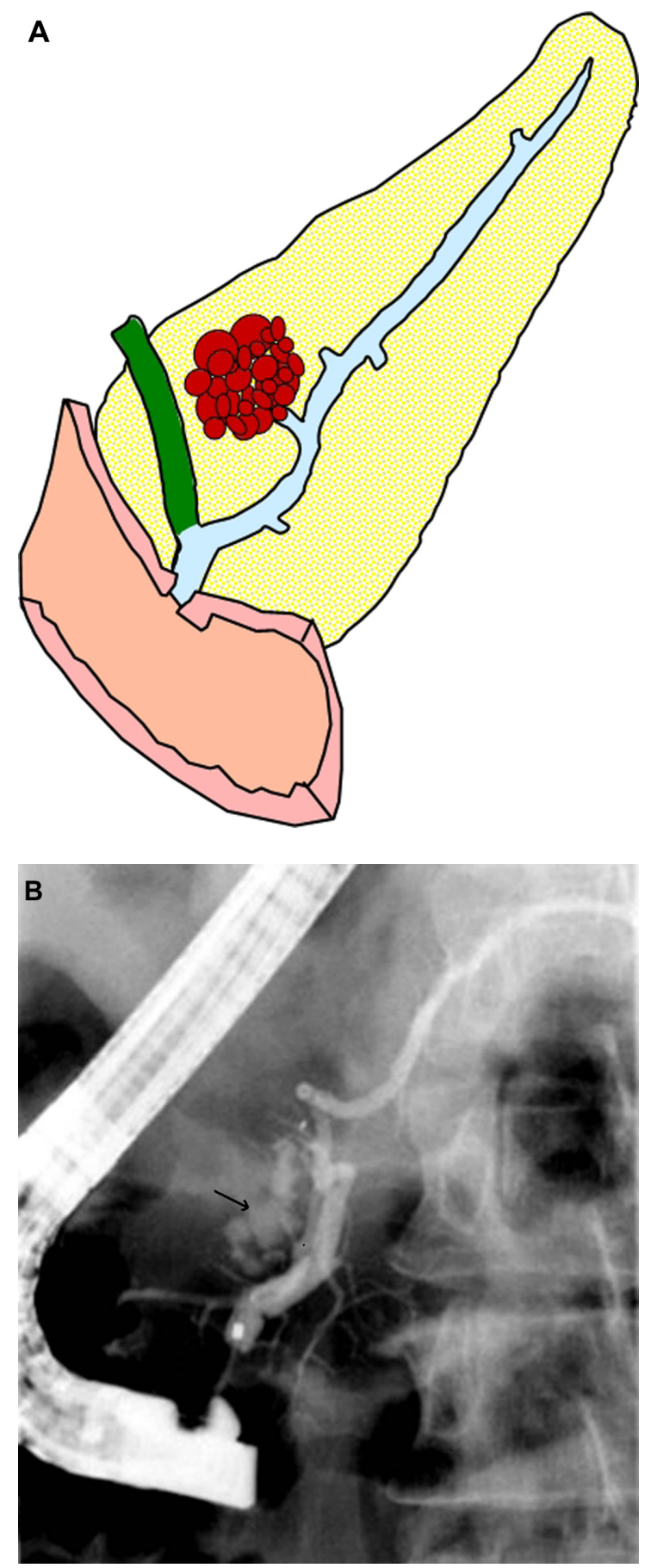

Figure 4 (A) Schematic of a branch-duct IPMN lesion arising from a ductal side-branch (appearance of a "bunch of grapes"). (B) Endoscopic retrograde cholangiopancreatography diagnosing a branch-duct IPMN lesion. Pancreatogram reveals a communicating, "bunch of grapes" lesion off the ductal side-branch in the head of the gland (arrow).

Abbreviation: IPMN, intraductal papillary mucinous neoplasm.

evidence of MPD dilation are categorized as BD-IPMNs. Radiographically, they often appear as a "bunch of grapes" growing from the end of a pancreatic ductal side-branch (Figure 4). IPMNs which meet criteria for both MD- and BD-IPMN are categorized as mixed type, which can have varying degrees of involvement with both the main duct and branch ducts. ${ }^{4,9}$

Definitive diagnosis of an IPMN is made based on the histology of resected cysts. Many BD-IPMNs exhibit some involvement with the main duct microscopically, and therefore grading these lesions in terms of the extent of main duct involvement may be a preferable approach, in contrast to categorizing all IPMNs as strictly MD-IPMN or BD-IPMN. ${ }^{4,9}$ Distinguishing between these subtypes is important, as MD-IPMNs are at increased risk for malignant transformation compared with BD-IPMNs. The prevalence of malignancy in resected MD-IPMN lesions ranges from $57 \%$ to $92 \%$, in contrast with $6 \%-46 \%$ for BD-IPMN lesions. ${ }^{4}$ Interestingly, on cyst fluid DNA analysis studies of IPMNs, the histological grade of dysplasia increases with the frequency of mutations in the $k$-ras gene. ${ }^{10}$ These findings suggest that $k$-ras gene mutations play a significant role in the process of carcinogenesis for these mucinous PCNs.

SPNs represent less than $10 \%$ of PCNs, and they occur predominantly in younger women, with a peak incidence ranging from the second to fourth decades of life. ${ }^{3}$ They are most commonly located in the body or tail of the pancreas, but the location is variable. Solid and cystic components may be present, as well as occasional calcifications within the cyst (Figure 5). Histologically, SPNs contain uniform cells with ovoid nuclei and eosinophilic granules, which are arranged in sheets. SPNs have a low potential for malignant transformation, but in general are considered to have a much higher risk for cancer compared with benign SCAs. These lesions have an excellent prognosis when completely resected, as the overall incidence of malignancy is estimated to be less than $15 \%$. Metastasis to sites including the liver, peritoneum, and lymph

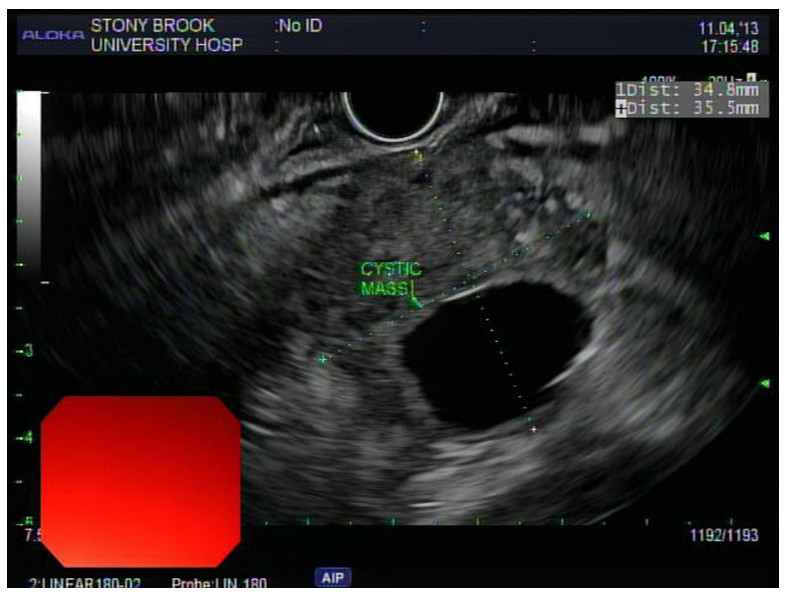

Figure 5 Endoscopic ultrasound image of a partially solid and partially cystic solid pseudopapillary neoplasm within the tail of the pancreas (dotted lines). The small black space in the center of the lesion is a fluid or cystic component. 
nodes has been reported. Even in malignant cases, prolonged survival has been shown in patients with residual disease after surgery, or in patients with unresectable tumors. ${ }^{3,11}$

One additional less common class of PCNs is the cystic neuroendocrine neoplasm. These cysts represent less than $10 \%$ of PCNs, and they occur with equal distribution among men and women. They are most often seen in the fifth and sixth decades of life. Their malignancy potential is similar to that of solid neuroendocrine neoplasms. Appearance on crosssectional imaging is variable. Cytology of these non-mucinous cysts shows small cells with scant cytoplasm and monomorphic nuclei with "salt-and-pepper" chromatin. ${ }^{3}$ Cystic neuroendocrine neoplasms are typically nonfunctioning tumors that have undergone cystic degeneration and can be difficult to distinguish from other PCNs on imaging alone. One study found this cystic degenerative form represented $10 \%$ of all pancreatic neuroendocrine tumors. ${ }^{12}$ Their location is most commonly in the body and tail of the pancreas. These lesions are generally indolent in behavior and carry a good prognosis; however, surgical resection should be considered in appropriate surgical candidates, given the premalignant nature of these lesions, particularly when they are larger than $2 \mathrm{~cm}$ in size..$^{12,13}$

\section{Clinical presentation}

Most pancreatic cysts are asymptomatic at the time of diagnosis, and they are often discovered incidentally when abdominal imaging is performed for evaluation of an unrelated problem. When PCNs are symptomatic, symptoms are typically the result of pancreaticobiliary duct obstruction. As such, clinical findings may include recurrent pancreatitis, chronic abdominal pain, or jaundice. Other nonspecific symptoms which may be present include nausea, vomiting, back pain, weight loss, or anorexia. The symptoms of advanced PCNs with malignant transformation may mimic those caused by pancreatic ductal adenocarcinoma (eg, jaundice, weight loss, and pain). Obstruction of the MPD (typically from mucin due to an IPMN, or compression of the duct by mass effect from the lesion) may present as acute or chronic pancreatitis (Figure 6). Development of exocrine and endocrine pancreatic insufficiency is not uncommon, due to atrophy of the distal gland downstream of the obstruction.

PCNs and small pancreatic pseudocysts are often mistaken for one another given their similar presentations and imaging characteristics. The clinical context is often needed to help differentiate between PCNs and pseudocysts, as the latter are more likely to develop in the setting of prior pancreatitis (either recent or remote past), and are typically associated with pain. ${ }^{3,14}$ One study investigating 212 patients with pancreatic
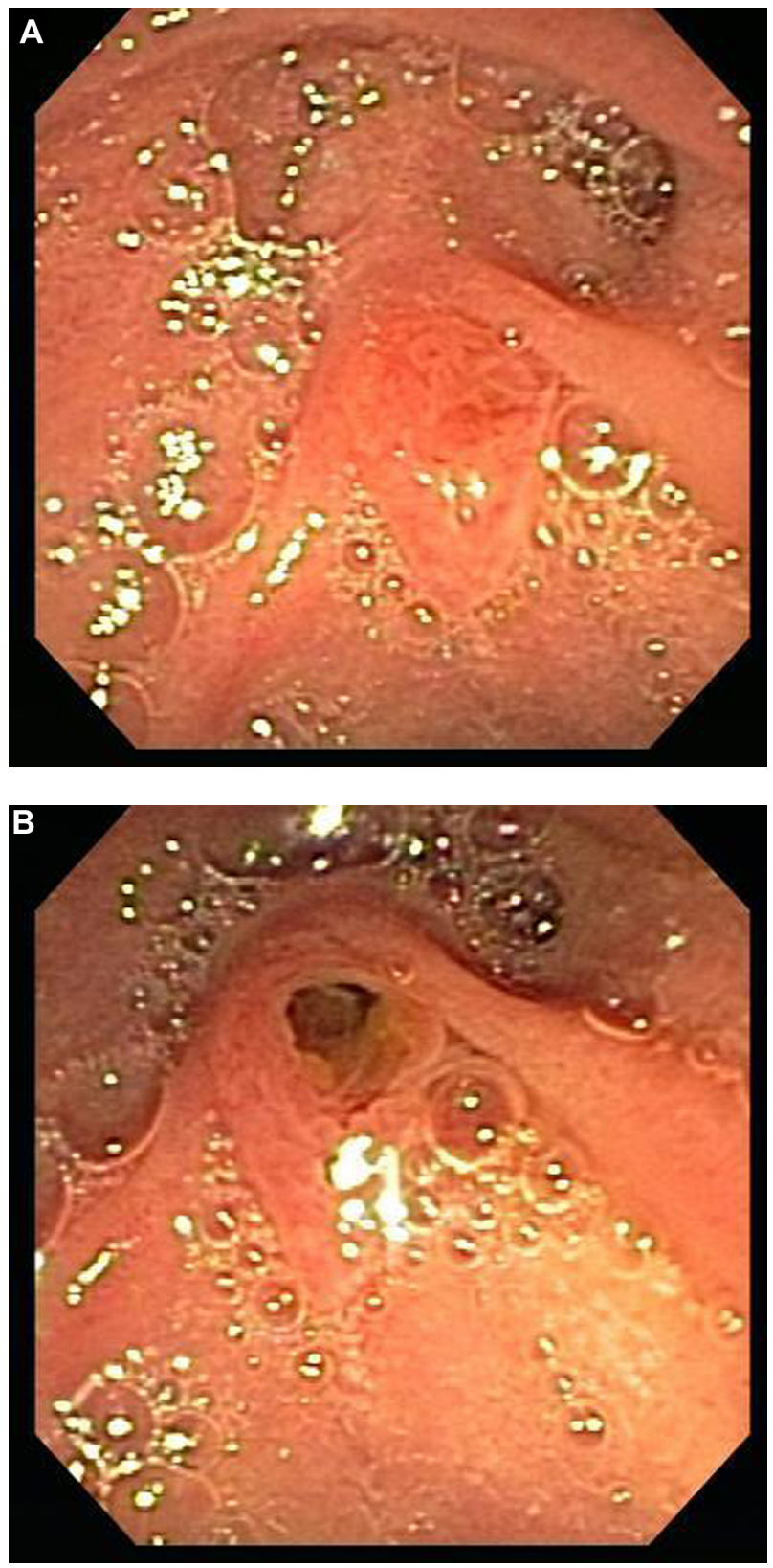

Figure 6 (A) Normal major papilla. (B) Major papilla with mucin extruding from the opening; investigated for recurrent acute pancreatitis in this patient.

cystic lesions in a surgical practice showed that $36.7 \%$ of the patients were asymptomatic. ${ }^{1}$ These asymptomatic cysts were more common in the elderly, smaller in size than symptomatic cysts, and less likely to be pseudocysts on final surgical pathology. Furthermore, greater than half of the asymptomatic cysts were found to be PCNs with dysplastic changes or malignant transformation. Another recent study followed 105 patients who underwent preoperative endoscopic ultrasound (EUS) for cyst evaluation. In this study, only $10 \%$ of patients were asymptomatic. Of the 70 patients with EUS cyst size less than $3 \mathrm{~cm}, 12$ patients (17\%) had a malignancy as diagnosed 
from EUS. ${ }^{15}$ The above studies support the role for periodic surveillance in all patients with PCNs, regardless of the size of the lesion or presence of symptoms.

\section{Diagnosis}

Pancreatic cystic lesions are often initially detected on crosssectional abdominal imaging such as CT or MRI. These modalities can help characterize the morphological features of pancreatic cysts such as cyst wall calcification, the presence of septae or mural nodules, and concurrent changes consistent with pancreatitis. In contrast to CT scan, MRI with magnetic resonance cholangiopancreatography (MRCP) serves to evaluate the pancreatic ductal system and often establishes the existence of ductal communication with a pancreatic cyst. According to a consensus of surveyed radiologists, the optimal procedure for evaluating pancreatic cystic lesions is a dedicated MRI-MRCP due to superior contrast resolution with better visualization of septae, nodules, and ductal communications. . $^{3,9,16}$

In another study evaluating the accuracy of CT versus MRI-MRCP in the characterization of IPMN disease, ductal connection was found on $73 \%$ of MRCP scans and only $18 \%$ of CT scans. ${ }^{17} \mathrm{CT}$ scans overestimated MPD involvement when compared with MRCP and surgical pathology. MRCP identified multifocal disease in $72 \%$ of cases versus $50 \%$ on CT. Additionally, MRCP was superior in visualization of branch duct lesions. ${ }^{17}$

Although PCNs often appear morphologically similar on cross-sectional imaging, particular characteristic features of specific PCN types can aid in diagnosis without further invasive testing. For example, the presence of a central scar seen on CT or MRI is highly suggestive of a serous lesion (SCA), and noted in roughly $20 \%$ of these cysts. For MCN lesions, the CT finding of peripheral "eggshell calcifications" (small calcifications on the periphery of macrocystic spaces within the lesion) is rare but strongly predictive of malignancy. Newer types of CT imaging, such as high-resolution multi-slice helical imaging, can more accurately identify potentially malignant features of IPMNs, such as the presence of mural nodules and segmental or diffuse dilatation of the MPD $>15 \mathrm{~mm}$ in diameter. Certain characteristics on $\mathrm{CT}$, in combination with clinical history, can distinguish true cysts from pseudocysts. Pseudocysts are more likely to be associated with findings of chronic pancreatitis such as gland atrophy, ductal dilatation, parenchymal calcification, and calculi in the pancreatic duct. ${ }^{3}$

When further evaluation of a PCN beyond routine crosssectional imaging is required, EUS with fine-needle aspiration (FNA) may be utilized. EUS-FNA allows for better imaging characterization of cyst morphology (eg, the presence of a mural nodule or a solid component), and it enables aspiration of cyst fluid for further analysis. Despite its unique ability to obtain high quality pancreatic imaging from within the lumen of the upper gastrointestinal tract, previous studies have suggested a limited ability of EUS alone to distinguish between benign and early dysplastic or malignant lesions, especially if features of frank malignancy are not present. ${ }^{3,16}$ Furthermore, the accurate interpretation of EUS findings is operator dependent and often varies from one endoscopist to another. One study investigated the ability of endosonographers to establish a diagnosis of a PCN, and also to determine the presence or absence of malignancy solely based on the EUS findings. ${ }^{18}$ Poor to fair agreement was observed between endosonographers in both tasks. Another study evaluated the accuracy of preoperative imaging with CT, endoscopic retrograde cholangiopancreatography (ERCP), or EUS in the detection of invasive versus noninvasive IPMN and MCN lesions. ${ }^{19}$ The overall accuracy for detecting invasion was less than $80 \%$ for all three diagnostic modalities.

ERCP enables inspection of the duodenal papilla for mucin extrusion, a finding which occurs in $20 \%-50 \%$ of main duct IPMNs, and is essentially pathognomonic for the disease. In addition, ERCP allows for the ability to perform pancreatography to assess communication of the cyst with the MPD. That said, with today's high quality (noninvasive) MRCP studies, there is little role for diagnostic ERCP in the work-up of a PCN. Due to the relatively high risk of post-procedural pancreatitis when performing ERCP of the pancreatic duct (20\%-30\%), this invasive procedure is most often reserved for patients in whom the diagnosis of main duct IPMN is highly suspected, and one is attempting to diagnose frank malignancy within the duct. The combination of intraductal pancreatoscopy with intraductal ultrasound at the time of ERCP can demonstrate malignancy with a high level of precision, and allow for accurate sampling of ductal nodules and other areas of concern. ${ }^{3,4,16}$ These procedures are generally performed only at specialized centers by expert interventional endoscopists.

Compared with ERCP, EUS evaluation with FNA is a less invasive and safer endoscopic procedure for the diagnosis of a PCN. Cyst fluid is obtained to assess for the presence of mucin, cytological atypia, carcinoembryonic antigen (CEA), and amylase levels, and DNA for molecular analysis. Studies have shown varying success in the accurate diagnosis of PCNs using cytology alone. However, identifying certain cell types using FNA can help narrow the diagnosis in certain instances. For example, the finding of glycogen-rich cuboidal cells suggests a diagnosis of SCA in the appropriate clinical setting; or the aspiration of inflammatory cells, such as macrophages and neutrophils, usually is suggestive of a pseudocyst..,9,16 For 
Table 2 Tumor markers in cyst fluid analysis

\begin{tabular}{lllll}
\hline Marker & \multicolumn{2}{l}{ Cyst type } & & \\
\cline { 2 - 5 } & IPMN & MCN & SCA & Pseudocyst \\
\hline CEA & High & High & Low & Variable \\
Amylase & High & Low & Low & High \\
CA 72-4 & High & High & Low & Variable \\
\hline
\end{tabular}

Abbreviations: CA, carbohydrate antigen; CEA, carcinoembryonic antigen; IPMN, intraductal papillary mucinous neoplasm; MCN, mucinous cystic neoplasm; SCA, serous cystadenoma.

the diagnosis of malignancy within PCNs, EUS-FNA with cytology alone is highly specific (approximately 90\%); yet, the sensitivity of EUS-FNA for a malignant PCN may be as low as $40 \%-50 \%$, with high false-negative rates.

In addition to cytology, the cyst fluid from PCNs can be used to measure levels of tumor markers and pancreatic enzymes (Table 2). CEA is a marker that has been shown to differentiate mucinous from non-mucinous cysts with $80 \%$ accuracy using levels $>192 \mathrm{ng} / \mathrm{mL}$ as a threshold suggestive of a mucinous lesion. ${ }^{3}$ However, a low CEA level $(<192 \mathrm{ng} / \mathrm{mL})$ does not fully exclude a mucinous cyst. Furthermore, CEA levels have not been shown to distinguish benign from malignant lesions. ${ }^{9,16}$ The presence of amylase in cyst fluid may suggest a communication between the PCN and the pancreatic ductal system, typically characteristic of an IPMN. However, elevated amylase levels are also found in pseudocysts. Low amylase concentrations in cyst fluid are associated with noncommunicating lesions such as SCAs and MCNs. ${ }^{3}$ Although the tumor marker carbohydrate antigen (CA) 19-9 has been shown to have an association with pancreatic adenocarcinoma, its presence in cyst fluid has not been helpful in distinguishing between mucinous and nonmucinous PCNs. ${ }^{3}$ The presence of CA 72-4 in cyst fluid has, however, been shown to be indicative of a mucinous lesion. One study showed that elevated serum CA 19-9 in combination with elevated CA 72-4 in cyst fluid is associated with mucinous neoplasms and ductal adenocarcinomas, and these patients should be considered for resection. ${ }^{3,20}$

Recent developments in DNA molecular analysis of cyst fluid have identified genes potentially associated with certain cyst types or PCNs. This can further help with the diagnosis of PCNs when cytological analysis is unrevealing due to scant cellularity in the cyst fluid, especially when a solid component is not present for sampling. A recent study showed that the presence of a $k$-ras gene mutation is diagnostic of a mucinous cyst. ${ }^{21}$ Furthermore, cyst fluid demonstrating large amounts of DNA, high-amplitude mutations, or a mutational sequence of k-ras mutation followed by allelic loss ("loss of heterozygosity”) is highly suspicious for malignancy. ${ }^{9,21}$
Another recent study demonstrated that GNAS gene mutations were found in $66 \%$ of IPMNs, and either GNAS or k-ras mutations were present in $96 \%$ of IPMNs. ${ }^{22}$ Other biomarkers that have been analyzed include micro-ribonucleic acid ( $\mathrm{miR}$ ). In one study, endoscopically acquired pancreatic cystic fluid was obtained from 38 patients who subsequently had surgical resection of the cystic lesion. ${ }^{23}$ Levels of two specific miRs (miR-21 and miR-221) were found in higher concentrations in the malignant versus benign cystic lesions.

Overall, cyst fluid analysis is often times complex. Interpretation of the results can be difficult and variable depending upon the type of lesion, and the amount of fluid able to be aspirated at the time of the EUS procedure. The information obtained from the cyst fluid is used in conjunction with the patient's clinical presentation, as well as specific cyst-related morphological features on imaging, in order to make an overall assessment in terms of the type of $\mathrm{PCN}$ present and its inherent risk of malignancy. With the development of new and emerging molecular markers, cyst fluid analysis is likely to become even more complex in its attempt to risk-stratify specific PCNs. However, in the opinion of these authors, current data do not support the widespread use of molecular analysis in cyst fluid interpretation due to low overall specificity and sensitivity of the tests. For example, a recent study characterized the performance of molecular analysis (DNA) in diagnosing mucinous lesions. ${ }^{24}$ DNA analysis was performed on cyst fluid and compared with resection specimens. Molecular analysis had a sensitivity of $50 \%$ and specificity of $80 \%$ in identifying mucinous lesions. Diagnostic performance did increase when combined with CEA and cytology; however, the study clearly shows that currently available molecular analysis studies are insufficient when used alone. In routine clinical practice, we reserve molecular analysis of cyst fluid only for those select patients with "borderline" lesions in which we may be searching for more information to guide a patient toward, or away from, surgical resection (as opposed to continued surveillance).

\section{Management of PCNs}

The various diagnostic modalities discussed above can be useful for narrowing down the diagnosis of the exact type of PCN; however, definitive diagnosis is often times difficult without supporting histological evidence (ie, by means of surgical resection). Given this diagnostic challenge, a more practical approach to risk-stratification has been suggested and outlined in a recent paper by Tanaka et al. ${ }^{9}$ Within this publication, evidence-based guidelines were devised by expert physicians and surgeons of the International Association of Pancreatology (IAP). The guidelines risk-stratify 
PCNs based on "worrisome features" and "high-risk stigmata" to determine management of these lesions based on their malignant potential. Furthermore, they outline the stepwise use of multiple imaging modalities when further work-up is required in order to determine whether a PCN is appropriate for surgical resection.

The evidence-based "worrisome features" based on multiple imaging modalities include cyst size $\geq 3 \mathrm{~cm}$, thickening or hyperenhancement of the cyst walls, MPD size of 5-9 mm, mural nodules, abrupt change in the MPD caliber (with distal pancreatic atrophy), and regional lymphadenopathy. Features on CT, MRI, or EUS such as an obstructed common bile duct in a patient with a lesion of the pancreatic head, an enhanced solid component to the cyst, and MPD size $\geq 10 \mathrm{~mm}$ are highly suspicious for malignancy and are thus termed "high-risk stigmata" (Table 3). ${ }^{9}$ Patients who demonstrate "high-risk stigmata" should be strongly considered for surgical resection unless clinically contraindicated due to high operative risk. Furthermore, PCNs that cause symptoms (eg, abdominal pain, pancreatitis, and weight loss) often necessitate strong consideration for surgical resection, as the presence of symptoms has been shown to confer a higher risk of malignant transformation. ${ }^{9}$

The following algorithm for risk-stratification and management of PCNs has been proposed: any PCN with features showing "high-risk stigmata" should be considered for surgical resection if no clinical contraindications exist. If "high-risk stigmata" are not present on noninvasive imaging studies, the next step is to assess for "worrisome features." If "worrisome features" are suggested on CT or MRI, an EUS examination should be performed by an experienced endoscopist to assess for these features, looking specifically for the presence of a

Table 3 Moderate and high-risk features of mucinous PCNs (ie, IPMN and MCN lesions)

\begin{tabular}{ll}
$\begin{array}{l}\text { “Worrisome features" } \\
\text { (moderate-risk) }\end{array}$ & $\begin{array}{l}\text { “High-risk stigmata” } \\
\text { (high-risk) }\end{array}$ \\
\hline Clinical evidence of pancreatitis & $\begin{array}{l}\text { Obstructed common bile } \\
\text { duct associated with lesion } \\
\text { of the pancreatic head } \\
\text { Enhanced solid component } \\
\text { within the cyst } \\
\text { Cyst size }>3 \mathrm{~cm}\end{array}$ \\
$\begin{array}{l}\text { Thickened/enhanced cyst walls } \\
\geq 10 \text { mm }\end{array}$ \\
$\begin{array}{l}\text { Main pancreatic duct size 5-9 mm } \\
\text { Non-enhanced mural nodules }\end{array}$ \\
$\begin{array}{l}\text { Abrupt change in main pancreatic duct } \\
\text { caliber, with distal pancreatic atrophy } \\
\text { Regional lymphadenopathy }\end{array}$ \\
\hline
\end{tabular}

Abbreviations: IPMN, intraductal papillary mucinous neoplasm; $M C N$, mucinous cyst adenoma; PCN, pancreatic cystic neoplasm. mural nodule, MPD abnormalities with gland atrophy, thickened or enhanced portions of the cyst wall, and the presence of undetected regional lymphadenopathy. In addition, EUS-FNA may be performed to obtain cyst fluid for cytological analysis which may be suspicious or positive for malignancy. Based on these findings surgery may be considered.

If none of these "worrisome features" are present, different intervals of surveillance should occur based on the cyst size. For cyst size $2-3 \mathrm{~cm}$, patients should be evaluated with MRI or EUS as frequently as every 3-6 months, with consideration for resection in young, surgically fit individuals. For cyst size 1-2 cm, monitoring with CT or MRI annually may be considered, with lengthening of the surveillance interval if no changes in cyst features are present. For small lesions $<1 \mathrm{~cm}$ in size, monitoring with $\mathrm{CT}$ or MRI can be performed every $2-3$ years. $^{9}$

Management of suspected IPMN lesions differs depending on the origination of the cystic lesion within the main duct or the branch ducts. According to a published series, main duct IPMNs have a mean frequency of malignancy of $61.6 \%$, and a mean frequency of invasive disease of $43.1 \%{ }^{4,9}$ In one series, the three factors most predictive of malignancy in MD-IPMN and mixed-type IPMN lesions were the presence of symptoms, mural nodules, and MPD diameter $15 \mathrm{~mm}$ or greater. ${ }^{4,25}$ Another large study of resected MD-IPMNs showed malignancy was associated with older age (at least 6 years older than their benign counterparts), as well as the presence of jaundice or worsening diabetes at presentation. ${ }^{4,26}$ Several patients, however, in both series had no obvious clinical or radiographic predictors of advanced disease, yet were found to have malignancy at the time of resection. Given the high prevalence of malignancy in MD-IPMN lesions, it is inferred that most MD-IPMNs go on to progress to malignancy. Coupled with the low overall 5-year survival rates following surgical resection (31\%-54\%), guidelines suggest removal of all MD-IPMNs in surgically fit patients with the aim of complete resection of the cyst with negative margins. Long-term follow-up of patients with resected noninvasive MD-IPMNs has shown good longterm survival rates. Resection of invasive IPMNs results in a 5 -year survival rate ranging from $36 \%$ to $60 \%{ }^{4,9}$

The management of branch duct IPMNs is less clear and depends greatly on the clinical context. The malignant potential of a BD-IPMN is less than MD-IPMN, with a mean frequency of malignancy of $25.5 \%$, and a mean frequency of invasive cancer of $17.7 \% .{ }^{9}$ Sugiyama et al showed the two strongest predictors of malignancy in BD-IPMN lesions are the presence of a mural nodule and cyst size $>30 \mathrm{~mm} .{ }^{4,25}$ Furthermore, Matsumoto et al demonstrated that BD-IPMNs with size 
$<30 \mathrm{~mm}$ and the absence of mural nodules are highly unlikely to be malignant. ${ }^{27}$ These patients were followed for 33 months, and the majority remained asymptomatic without progression to advanced disease. In addition to size and the presence of mural nodules, a rapid rate of cyst growth is another high-risk factor. ${ }^{4,9}$ One study investigated BD-IPMNs $<30 \mathrm{~mm}$ and without mural nodules. ${ }^{28}$ During follow-up, $17.4 \%$ of the patients underwent resection, and the malignant cysts had grown by a greater percentage $(69.8 \%$ versus $19.4 \%)$, and at a greater rate (4.1 mm versus $1.0 \mathrm{~mm}$ per year), when compared with the nonmalignant cysts. Overall, a cyst growth rate of more than $2 \mathrm{~mm} /$ year was associated with a higher risk of malignancy. ${ }^{28}$ In addition, high-grade cellular atypia on EUS-FNA results, as opposed to "positive cytology," was also found to be a high-risk factor for malignancy in BD-IPMNs. ${ }^{9}$ The results of these studies and many others are the basis for the algorithmic guidelines suggested by Tanaka et al in regards to the management of BD-IPMN lesions. ${ }^{4,9}$ Given the lower risk for malignancy when compared with MD-IPMN lesions, conservative management with periodic surveillance is reasonable, particularly in older patients and those without worrisome features.

MCNs have an overall prevalence of invasive carcinoma of less than $15 \%{ }^{4,9}$ Malignancy is usually absent in MCNs $<3-4 \mathrm{~cm}$ in size. Since these lesions commonly occur in the body and tail of the pancreas, surgical resection is more often less invasive, since a distal pancreatectomy may be performed as opposed to pancreaticoduodenectomy. In younger patients with cysts located in the distal pancreas, surgery should be strongly considered in the presence of any worrisome features. The prognosis for patients undergoing resection of MCNs prior to the development of invasive disease is excellent. Once an MCN develops into a mucinous cystadenocarcinoma, resectability is difficult, which leads to poor prognosis. ${ }^{3,4,9}$

As described above, SCAs have an extremely low potential for malignant transformation. If the work-up of a $\mathrm{PCN}$ is strongly suggestive of an SCA, and the patient has no symptoms due to the cyst, these lesions can be managed conservatively with observation. Typically, repeat imaging is only needed if symptoms develop. If the diagnosis is unclear, or if the SCA causes symptoms (usually seen with SCA size $>4 \mathrm{~cm}^{29}$ ), surgical resection should be considered..$^{3,16}$

SPNs have a low - but significant - potential for malignancy. One study investigated a group of patients who underwent resection for pathologically confirmed SPNs. A total of $15 \%$ of the resected SPNs were malignant, without corresponding preoperative features predictive of malignancy. ${ }^{30}$ Given the low-grade potential for malignancy in SPN lesions, and the high cure rate if completely resected, the threshold for surgical resection in appropriate patients should be low; especially since most of these patients are young women below the age of 45 years old..$^{3,16}$

The type of resection depends on the location of the PCN and the extent of involvement. Limited resections can be considered for MCNs and BD-IPMNs without findings suspicious for malignancy or invasion. However, limited resections are technically difficult and associated with complications such as leaks and positive margins. Therefore, limited pancreatectomy should only be performed if negative margins can be definitively obtained. ${ }^{4}$ The standard treatment of any PCN with an invasive component is pancreaticoduodenectomy, distal pancreatectomy, or total pancreatectomy. In recent years, the mortality rate for pancreatic resection has fallen to less than $2 \%$ at high-volume centers. MCNs are typically located in the tail of the pancreas and can be resected using a distal pancreatectomy (with or without splenectomy). IPMNs are frequently located in the head and may require pancreaticoduodenectomy, or total pancreatectomy if more extensive involvement of the ductal system is discovered intraoperatively. ${ }^{3}$

Recent studies have examined nonsurgical methods for treating PCNs. EUS-guided mucosal ablation by ethanol injection into the cyst cavity is a novel technique that has been recently investigated. Ethanol induces cell membrane lysis and protein denaturation, which results in coagulative necrosis. Typical candidates for this investigative approach have been patients who are poor surgical candidates with worrisomeappearing cysts that lack communication with the MPD (so as not to inject alcohol directly into the pancreatic ductal system). The initial pilot study performed by Gan et $\mathrm{al}^{31}$ showed that ethanol ablation is safe and feasible, and a subset of patients ( 8 of 23 on follow-up) underwent complete resolution of the cyst. A follow-up study by DeWitt et $\mathrm{al}^{32}$ showed that EUSguided ethanol lavage resulted in a greater decrease in cyst size compared with saline lavage. Follow-up using CT surveillance revealed no cyst recurrence for a median of 26 months, and the percentage of complete pancreatic cyst ablation was 33\%. Studies have also investigated EUS-guided ethanol ablation followed by local injection of paclitaxel. ${ }^{33}$ Overall, these findings are promising and may present an alternative therapy for patients unwilling or unfit for surgery. However, it should be noted that complications such as pain and pancreatitis are relatively common. More research at high-volume centers is needed before EUS-guided ethanol ablation can be recommended to patients over surgical resection.

Several studies have investigated current trends in the evaluation and management of PCNs among physicians. In one recent study, a comparison of practice habits and awareness 
of consensus guidelines was examined between general gastroenterologists and surgeons, and a specialist group of EUS experts. ${ }^{34}$ Awareness of the existence of published guidelines for the diagnosis and management of PCNs was less common in the general group than the specialist group (64\% versus 33\% unaware, respectively). The American Society for Gastrointestinal Endoscopy guidelines were more commonly recognized by both groups, rather than the IAP guidelines. Both groups demonstrated only moderate consistency employing the published recommendations into their clinical practice.

\section{Follow-up}

Data for surveillance intervals of IPMNs and other PCNs are limited and depend largely on clinical judgment and the perceived risk for malignancy, comorbidities, and patient preference. Surveillance of non-resected IPMNs with EUS or MRI at appropriate intervals based on cyst size and other features as dictated by the IAP guidelines has been discussed above. ${ }^{9}$

Interval surveillance imaging after IPMN resection is strongly recommended, as multifocal disease is common and additional lesions may develop in the remnant pancreas. Recurrence rates of new IPMN lesions following resection range from $0 \%-20 \%{ }^{4,9,35}$ In patients with noninvasive disease that was completely resected, at least an annual examination of the remnant pancreas with MRI or EUS is encouraged. However, the risk of developing invasive disease in another IPMN lesion within the gland appears to be very low. ${ }^{35}$ Surveillance for invasive IPMNs after resection should mimic follow-up guidelines for pancreatic ductal adenocarcinoma. For MCNs, given the nearly $100 \%$ cure rate following resection of noninvasive lesions, continued surveillance is unnecessary in most cases. Malignant MCNs should be followed frequently at 6-12 month intervals with either CT or MRI. ${ }^{4,9}$ Data for surveillance guidelines for the other PCN types is limited, and surveillance should be considered on an individual basis.

\section{Summary}

The increasing discovery of PCNs is largely due to the widespread use of new, cross-sectional imaging techniques. Physicians and surgeons need to be aware of the different types of pancreatic cysts so that a determination may be made regarding the potential for malignant transformation. Appropriate evaluation of a possible PCN includes a multidisciplinary approach among abdominal radiologists, gastroenterologists with a special expertise in EUS, and pancreatic surgeons. Updated published guidelines exist to help providers recognize higher risk lesions, and provide recommendations in terms of surveillance strategies and the need for possible pancreatic resection. Much is still unknown about PCNs, yet our knowledge on risk-stratification, optimal surveillance intervals, and post-surgical management is rapidly increasing.

\section{Disclosure}

The authors have no conflicts of interest in this work.

\section{References}

1. Fernandez-del Castillo C, Targarona J, Thayer SP, et al. Incidental pancreatic cysts: clinicopathologic characteristics and comparison with symptomatic patients. Arch Surg. 2003;138(4):427-433.

2. Hamilton SR, Aaltonen LA, editors. Pathology and genetics of tumours of the digestive system. In: Vol 2 of World Health Organization Classification of Tumours. Lyon, France: IARC Press; 2000.

3. Brugge WR, Lauwers GY, Sahani D, Fernandez-del Castillo C, Warshaw AL. Cystic neoplasms of the pancreas. $N$ Engl J Med. 2004;351:1218-1226.

4. Tanaka M, Chari S, Adsay V, et al. International consensus guidelines for management of intraductal papillary mucinous neoplasms and mucinous cystic neoplasms of the pancreas. Pancreatology. 2006;6: 17-32.

5. Galanis C, Zamani A, Cameron JL, et al. Resected serous cystic neoplasms of the pancreas: A review of 158 patients with recommendations for treatment. $J$ Gastrointest Surg. 2007;11(7):820-826.

6. King JC, Ng TT, White SC, Cortina G, Reber HA, Hines OJ. Pancreatic serous cystadenocarcinoma: a case report and review of the literature. $J$ Gastrointest Surg. 2009;13(10):1864-1868.

7. Mohr VH, Vortmeyer AO, Zhuang Z, et al. Histopathology and molecular genetics of multiple cysts and microcystic (serous) adenomas of the pancreas in von Hippel-Lindau patients. Am J Pathol. 2000;157: $1615-1621$

8. Vortmeyer AO, Lubensky IA, Fogt F, Linehan WM, Khettry U, Zhuang Z. Allelic deletion and mutation of the von Hippel-Lindau (VHL) tumor suppressor gene in pancreatic microcystic adenomas. Am J Pathol. 1997; 151:951-956.

9. Tanaka M, Fernandez-del Castillo C, Adsay V, et al. International consensus guidelines 2012 for the management of IPMN and MCN of the pancreas. Pancreatology. 2012;12:183-197.

10. Z'graggen K, Rivera JA, Compton CC, et al. Prevalence of activating $\mathrm{K}$-ras mutations in the evolutionary stages of neoplasia in intraductal papillary mucinous tumors of the pancreas. Ann Surg. 1997;226: 491-498.

11. Ng KH, Tan PH, Thng CH, Ooi LL. Solid pseudopapillary tumour of the pancreas. ANZ J Surg. 2003;73(6):410-415.

12. Ahrendt SA, Komorowski RA, Demeure MJ, Wilson SD, Pitt HA. Cystic pancreatic neuroendocrine tumors: is preoperative diagnosis possible? J Gastrointest Surg. 2002;6:66-74.

13. Buetow PC, Parrino TV, Buck JL, et al. Islet cell tumors of the pancreas: pathologic-imaging correlation among size, necrosis and cysts, calcification, malignant behavior, and functional status. AJR Am J Roentgenol. 1995; 165:1175-1179.

14. Bassi C, Procacci C, Zamboni G, et al. Intraductal papillary mucinous tumors of the pancreas. Verona University Pancreatic Team. Int J Pancreatol. 2000;27:181-193.

15. Wong J, Weber J, Centeno BA, et al. High grade dysplasia and adenocarcinoma are frequent in side-branch intraductal papillary mucinous neoplasm measuring less than $3 \mathrm{~cm}$ on endoscopic ultrasound. J Gastrointest Surg. 2013;17(1):78-84.

16. Khalid A, Brugge W. ACG practice guidelines for the diagnosis and management of neoplastic pancreatic cysts. Am J Gastroenterol. 2007;102:2339-2349

17. Waters JA, Schmidt CM, Pinchot JW, et al. CT vs MRCP: optimal classification of IPMN type and extent. J Gastrointest Surg. 2008;12(1): 101-109. 
18. Ahmad NA, Kochman ML, Brensinger C, et al. Interobserver agreement among endosonographers for the diagnosis of neoplastic versus nonneoplastic pancreatic cystic lesions. Gastrointest Endosc. 2003;58: 59-64.

19. Cellier C, Cuillerier E, Palazzo L, et al. Intraductal papillary and mucinous tumors of the pancreas: Accuracy of preoperative computed tomography, endoscopic retrograde pancreatography, and endoscopic ultrasonography, and long-term outcome in a large surgical series. Gastrointest Endosc. 1998;47:42-49.

20. Sperti C, Pasquali C, Guolo P, Polverosi R, Liessi G, Pedrazzoli S Serum tumor markers and cyst fluid analysis are useful for the diagnosis of pancreatic cystic tumors. Cancer. 1996;78:237-243.

21. Khalid A, Zahid M, Finkelstein SD, et al. Pancreatic cyst fluid DNA analysis in evaluating pancreatic cysts: a report of the PANDA study. Gastrointest Endosc. 2009;69:1095-1102.

22. Wu J, Matthaei H, Maitra A, et al. Recurrent GNAS mutations define an unexpected pathway for pancreatic cyst development. Sci Transl Med. 2011;3(92):92ra66.

23. Farrell JJ, Toste $\mathrm{P}, \mathrm{Wu} \mathrm{N}$, et al. Endoscopically acquired pancreatic cyst fluid microRNA 21 and 221 are associated with invasive cancer. Am J Gastroenterol. 2013;108(8):1352-1359.

24. Al-Haddad M, Dewitt J, Sherman S, et al. Performance characteristics of molecular (DNA) analysis for the diagnosis of mucinous pancreatic cysts. Gastrointest Endosc. 2014;79(1):79-87.

25. Sugiyama M, Izumisato Y, Abe N, Masaki T, Mori T, Atomi Y. Predictive factors for malignancy in intraductal papillary-mucinous tumors of the pancreas. Br J Surg. 2003;90:1244-1249.

26. Salvia R, Fernandez-del Castillo C, Bassi C, et al. Main duct intraductal papillary mucinous neoplasms of the pancreas: clinical predictors of malignancy and long-term survival following resection. Ann Surg. 2004;239:678-687.
27. Matsumoto T, Aramaki M, Yada K, et al. Optimal management of the branch duct type intraductal papillary mucinous neoplasms of the pancreas. J Clin Gastroenterol. 2003;36:261-265.

28. Kang MJ, Jang JY, Kim SJ, et al. Cyst growth rate predicts malignancy in patients with branch duct intraductal papillary mucinous neoplasms. Clin Gastroenterol Hepatol. 2011;9:87-93.

29. Tseng JF, Warshaw AL, Sahani DV, Lauwers GY, Rattner DW, Fernandez del-Castillo C. Serous cystadenoma of the pancreas: Tumor growth rates and recommendations for treatment. Ann Surg. 2005;242: 413-421.

30. Lee SE, Jang JY, Hwang DW, et al. Clinical features and outcome of solid pseudopapillary neoplasm: differences between adults and children. Arch Surg. 2008;143:1218-1221.

31. Gan S1, Thompson CC, Lauwers GY, Bounds BC, Brugge WR. Ethanol lavage of pancreatic cystic lesions: initial pilot study. Gastrointest Endosc. 2005;61:746-752.

32. DeWitt J, McGreevy K, Schmidt CM, Brugge WR. EUS-guided ethanol versus saline solution lavage for pancreatic cysts: a randomized, doubleblind study. Gastrointest Endosc. 2009;70:710-723.

33. Oh HC, Seo DW, Song TJ, et al. Endoscopic ultrasonography-guided ethanol lavage with paclitaxel injection to treat patients with pancreatic cysts. Gastroenterology. 2011;140(1):172-179.

34. Buscaglia JM, Shin EJ, Giday SA, et al. Awareness of guidelines and trends in the management of suspected pancreatic cystic neoplasms: survey results among general gastroenterologists and EUS specialists. Gastrointest Endosc. 2009;69:813-820.

35. Miller JR, Meyer JE, Walters JA, et al. Outcome of the pancreatic remnant following pancreatectomy for non-invasive intraductal papillary mucinous neoplasm. HPB (Oxford). 2011;13(1):759-766.
Journal of Multidisciplinary Healthcare

\section{Publish your work in this journal}

The Journal of Multidisciplinary Healthcare is an international, peerreviewed open-access journal that aims to represent and publish research in healthcare areas delivered by practitioners of different disciplines. This includes studies and reviews conducted by multidisciplinary teams as well as research which evaluates the results or conduct of such teams or

\section{Dovepress}

healthcare processes in general. The journal covers a wide range of areas and welcomes submission from practitioners at all levels, from all over the world. The manuscript management system is completely online and includes a very quick and fair peer-review system. Visit http://www.dovepress.com/testimonials.php to read real quotes from published authors. 